\title{
Dermestes maculatus: an intermediate-germ beetle model system for evo-devo
}

Jie Xiang ${ }^{1,2}$, lain S. Forrest ${ }^{1}$ and Leslie Pick ${ }^{1,2^{*}}$

\begin{abstract}
Background: Understanding how genes change during evolution to direct the development of diverse body plans is a major goal of the evo-devo field. Achieving this will require the establishment of new model systems that represent key points in phylogeny. These new model systems must be amenable to laboratory culture, and molecular and functional approaches should be feasible. To date, studies of insects have been best represented by the model system Drosophila melanogaster. Given the enormous diversity represented by insect taxa, comparative studies within this clade will provide a wealth of information about the evolutionary potential and trajectories of alternative developmental strategies.
\end{abstract}

Results: Here we established the beetle Dermestes maculatus, a member of the speciose clade Coleoptera, as a new insect model system. We have maintained a continuously breeding culture in the lab and documented Dermestes maculatus embryogenesis using nuclear and phalloidin staining. Anterior segments are specified during the blastoderm stage before gastrulation, and posterior segments are added sequentially during germ band elongation. We isolated and studied the expression and function of the pair-rule segmentation gene paired in Dermestes maculatus. In this species, paired is expressed in stripes during both blastoderm and germ band stages: four primary stripes arise prior to gastrulation, confirming an intermediate-germ mode of development for this species. As in other insects, these primary stripes then split into secondary stripes. To study gene function, we established both embryonic and parental RNAi. Knockdown of Dmac-paired with either method resulted in pair-rule-like segmentation defects, including loss of Engrailed expression in alternate stripes.

Conclusions: These studies establish basic approaches necessary to use Dermestes maculatus as a model system. Methods are now available for use of this intermediate-germ insect for future studies of the evolution of regulatory networks controlling insect segmentation, as well as of other processes in development and homeostasis. Consistent with the role of paired in long-germ Drosophila and shorter-germ Tribolium, paired functions as a pair-rule segmentation gene in Dermestes maculatus. Thus, paired retains pair-rule function in insects with different modes of segment addition.

Keywords: Evo-devo, Segmentation, Pair-rule patterning, paired, RNAi, Dermestes maculatus

\section{Background}

Understanding the basis for the diversity of plant and animal systems on our planet will require studies of the mechanistic basis of body patterning and developmental

\footnotetext{
*Correspondence: Ipick@umd.edu

${ }^{1}$ Department of Entomology, University of Maryland, 4112 Plant Sciences Building, College Park, MD 20742, USA

Full list of author information is available at the end of the article
}

strategies used in different species as well as an understanding of how these mechanisms evolved (evo-devo). It is crucial that these studies include sampling of species from a broad range of taxa that represent distinct branches of the tree of life (reviewed in [1]). Rapid progress in the development of genomic technologies has made it possible to readily identify genes in diverse species. However, understanding how these genes control 
developmental processes will require establishment of model systems in which gene function can be assessed.

Arthropods represent $\sim 80 \%$ of all described species; among them, insects are the dominant taxa, representing $\sim 65 \%$ of all animal species on the planet [2]. Insects are easy to experimentally manipulate, can often be readily cultured in the laboratory, producing large numbers of embryos with reasonable generation time, and their enormous diversity makes them an ideal group for comparative studies to probe phenotypic diversity and unravel ancestral mechanisms. Among insects, the most sophisticated model system available to date is Drosophila melanogaster (D. melanogaster). D. melanogaster serves as a reference species for any study of insects, or other new animal model, with more than 100 years of study by thousands of researchers throughout the world, a plethora of genetic tools to assess gene function, and progress on every type of 'omics' analysis [3]. D. melanogaster is a member of the group of holometabolous insects thought to have arisen 300-400 million years ago (Mya) [4], which includes $>80 \%$ of all extant insect species [5]. Additional models are needed from this group to understand diversity in Holometabola. The most speciose order of holometabolous insects is Coleoptera (beetles), with $>350,000$ named species representing $\sim 40 \%$ of all insect species $[2,6,7]$. Coleoptera are thought to have arisen $\sim 285$ Mya [8] and have radiated to occupy a broad variety of niches on our planet including those with extreme environments, such as the Arctic, high mountain altitudes and dry, desert terrains. Beetles range in size from $<0.5 \mathrm{~mm}$ to $>15 \mathrm{~cm}$ in length and feed on everything from other insects, to fungus, decaying wood, a wide variety of plants, animal debris and even dung. The most sophisticated coleopteran model system developed to date is the flour beetle, Tribolium castaneum (T. castaneum; [9-11]), providing a frame of reference for the development of additional beetle systems to represent the diversity of this large clade.

Segmentation is a highly conserved feature shared by arthropods and outgroups [12-16]. Despite this similarity, the ways in which segments form and the genes that control this process vary among taxa [17-19]. Krause first classified insect embryogenesis into short-, intermediate- and long-germ modes based on the relative size of the germ anlage prior to gastrulation [20]. These different modes of segmentation can be distinguished by the number of segments established in the germ rudiment before gastrulation: long-germ (all or most segments are established more or less simultaneously), short-germ (only anterior segments are specified) and intermediategerm (head and thorax segments, and sometimes anterior abdominal segments, are specified). Both short- and intermediate-germ insects differ from long-germ insects in that posterior segments are added sequentially from a posterior segment addition zone (SAZ) or growth zone. This strategy of 'sequential addition' of segments is thought to be ancestral to arthropods and it is only in holometabolous insects that long-germ development has been observed [21]. Phylogenetic studies and accumulating molecular evidence indicate that long-germ development in different orders of Holometabola has evolved independently $[17,22]$. How modes of segment formation switched without disrupting the segmented body plan itself is unclear. The presence of nurse cells, enlarged germ size, acquisition of an anterior patterning center, shifted gap gene expression boundaries, and diminished activity of a segmentation clock have been proposed as prerequisites for long-germ development [17-19, 22-24]. Studies of the mechanisms underlying segmentation in an intermediate-germ insect, which may reflect an intermediate state between short- and long-germ modes of segmentation, will yield information on the transition from ancestral sequential specification to long-germ development. In addition, since long-germ development appears to have evolved several times independently within Holometabola, it will be of interest to compare mechanisms in species within a single clade rather than just comparing all sequentially segmenting species to $D$. melanogaster. These comparative studies will distinguish stages in the evolution of the long-germ mode which may have been gradual, with increasing numbers of segments specified simultaneously in different species, or may have occurred in a punctuated fashion, reflecting developmental constraints that remain to be discovered.

The two best-developed insect systems, D. melanogaster and T. castaneum, represent different modes of segment addition with $D$. melanogaster displaying the long-germ mode and $T$. castaneum specifying segments sequentially. Genetic screens in D. melanogaster identified a group of pair-rule segmentation genes (PRGs) that control the formation of body segments, and many of these also function in segmentation in $T$. castaneum [25-27]. However, their specific roles in the segmentation process often differ and some genes involved in segmentation in D. melanogaster do not function in segmentation in T. castaneum $[25,28]$. Work from other insects suggests that new genes may be recruited into PRG networks and that PRG orthologs have acquired novel function in different lineages [29, 30]. To understand the extent to which mechanisms regulating segmentation vary, the genetic underpinnings of this process must be examined in different species. As first pointed out by Patel and Davis, Coleoptera are an ideal order for this comparison, as short-, intermediate- and longgerm development have all been observed in beetles [17, 31,32 . Comparison of gene function in species within 
the same clade displaying these different developmental strategies will provide information about the extent of variation among segmentation regulatory networks, the impact of these changes on downstream targets, and clues about how changes in gene expression and function drive the evolution of alternative developmental modes.

Here we have established Dermestes maculatus ( $D$. maculatus) as a system for comparative studies within Coleoptera. T. castaneum and D. maculatus diverged close to the time of origin of this clade 250 Mya, [33], making this pair of species ideal for comparative studies, as they represent divergent lineages within the order Coleoptera. D. maculatus display an intermediate-germ mode of segmentation compared to the shorter-germ mode of T. castaneum. D. maculatus are easy to rear in the lab, with high fecundity and a short life cycle. We characterized the early steps of nuclear division in $D$. maculatus embryos and isolated an ortholog of the $D$. melanogaster PRG, paired (prd). Dmac-prd has pair-rulelike expression and function, regulating the expression of alternate stripes of the segment polarity gene engrailed (en). These studies support the conclusion that the function of prd as a PRG is highly conserved across holometabolous taxa. Additionally, these studies establish methods for in situ hybridization, antibody staining, and both parental and embryonic RNAi in D. maculatus.

\section{Methods}

\section{Dermestes species verification using DNA barcoding}

D. maculatus adults and larvae were purchased from Carolina Biological Supply Company. To verify the identity of the species, we amplified the mitochondrial cytochrome c oxidase subunit I (COI) gene [34, 35]. Genomic DNA was extracted using a DNeasy Tissue kit (Qiagen). Only wings and legs were taken from four Dermestes adults to avoid contamination by gut content. PCR using the primer pair LCO1490 (5'-GGTCAACAAATCATAAAGATATTGG-3') and HCO2198 (5' ${ }^{\prime}$-TAAACT TCAGGG TGACCAAAAAATCA-3') amplified an approximately 700 base pair (bp) fragment. The sequence of this fragment matched $D$. maculatus COI (GenBank ID HM909035.1) except at position 581 (C to $\mathrm{T}$ transition, Additional file 1).

\section{Rearing of $D$. maculatus}

D. maculatus were kept in large plastic cages (14.5 in. long $\times 8.5 \mathrm{in}$. wide $\times 10 \mathrm{in}$. high) with a thin layer of wood shavings spread on the bottom. The beetles were fed cat food (Fancy Feast) placed in a small weigh boat and changed twice a week. No water was added to avoid fungal growth. As immobile final instar larvae and pupae would be slaughtered by younger larvae, chunks of styrofoam were placed in the cages for the larvae to crawl into and hide before eclosion. Mesh cloth was used to cover the cages to prevent beetle escape while keeping the cages well ventilated. Cages were placed in incubators at 25 or $30{ }^{\circ} \mathrm{C}$ for colony maintenance. To collect embryos, newly eclosed $D$. maculatus were selected from the colony and placed in small plastic cages (9 inches long $\times 6$ inches wide $\times 6.5$ inches high) without wood shavings. They were fed daily to provide sufficient food. Cotton balls were stretched out and placed in the cage for egg laying. The cages were held at either 25 or $30^{\circ} \mathrm{C}$ for developmental staging.

\section{Embryo collection and fixation}

The protocol for fixation of $D$. maculatus embryos was modified from standard D. melanogaster and Oncopeltus fasciatus (O. fasciatus) embryo fixation protocols $[36,37]$ as follows. Cotton balls were carefully torn apart to let embryos fall onto a black sheet of paper. Embryos are white, approximately $0.2 \mathrm{~cm}$ in length, and can be seen easily against the black background. Embryos were transferred into small beakers and treated with $50 \%$ bleach for $4 \mathrm{~min}$ followed by several water rinses. Embryos were then transferred into $1.5 \mathrm{ml}$ Eppendorf tubes with distilled $\mathrm{H}_{2} \mathrm{O}$ (approximately $200 \mu \mathrm{l}$ of embryos in $1000 \mu \mathrm{l}$ of distilled $\mathrm{H}_{2} \mathrm{O}$ ). Tubes were placed in boiling water for $3 \mathrm{~min}$ and then on ice for $7 \mathrm{~min}$ to swell the eggshell, making embryos easier to dissect before staining. Embryos were then fixed in heptane: 4 \% PFA 1:1 for $20 \mathrm{~min}$ on a shaker at high speed ( $250 \mathrm{rpm})$. PFA (lower phase) was removed and $\mathrm{MeOH}$ was added and the tube was shaken vigorously for $20 \mathrm{~s}$. After several $\mathrm{MeOH}$ washes, embryos were stored at $-20{ }^{\circ} \mathrm{C}$ in $\mathrm{MeOH}$. A detailed D. maculatus embryo fixation protocol is provided (Additional file 2).

\section{prd gene cloning and identification}

To isolate prd from $D$. maculatus embryonic mRNA, total RNA was extracted from 0 to 1 day (0-1 day) after egg laying (AEL) embryos developing at $30{ }^{\circ} \mathrm{C}$ using TRIzol (Invitrogen) and an RNeasy mini kit (Qiagen). Reverse transcription was performed using the QuantiTect Reverse Transcription kit (Qiagen) to prepare 0-1 day embryonic cDNA. Two rounds of degenerate PCR were performed (forward outer primer: prd-deg1 F: 5'-GGNGGNGTNTTYATHAAYGG-3', GGVFING; reverse outer primer: prd-deg1 R: $5^{\prime}$-RTTNSWRAACCANACYTG-3', QVWFSN; forward inner primer: prddeg2 F: 5'-MARATHGTNGARATGGC-3', KIVEMA; reverse inner primer: prd-deg2 R: 5'-RTANACRTCNGGRTAYTG-3', QYPDIY; [38]), generating a product of approximately 600 bp length. After purification and insertion into pGEM-T Easy Vector (Promega) by TA cloning, sequencing of individual clones revealed partial Dmac-prd, as well as partial sequences of the 
Pax3/7 family genes Dmac-gooseberry (gsb) and Dmacgooseberry-neuro $(g s b-n)$ [39-41]. The $3^{\prime}$ end of the Dmac-prd coding sequence and $3^{\prime}$ UTR were isolated through two rounds of $3^{\prime} \mathrm{RACE}$ using gene-specific primers and the FirstChoice RLM-RACE kit (Ambion) following the manufacturer's instructions (1st round outer primer: AGAAACAGGCTCGATTCGTC, 1st round inner primer GATCGTCTCGTCAAGGAAGG; 2nd round outer primer: $5^{\prime}$ TTAGCTGGTGGCATTCAAAA, 2nd round inner primer $5^{\prime}$ AAGCTCTGTTGGTGCTGGTT). A contiguous fragment spanning part of the paired domain (PD) through the stop codon was verified using gene-specific primers: Dmac-prd3'F $5^{\prime}$ AGAAACAGGCTCGATTCGTC and Dmac-prd $3^{\prime} \mathrm{R} 5^{\prime}$ CAGTTGGGTAACTCAGTGAACG. The region coding for the $\mathrm{C}$-terminus of the PD through the stop codon was inserted into the XhoI and XbaI restriction sites of a KS vector for use as template for RNA in situ hybridization probe and double-stranded RNA (dsRNA) syntheses (KS-Dmac-prd).

\section{Embryo developmental staging, RNA in situ hybridization and antibody staining}

For D. maculatus developmental staging, embryos were collected every $2 \mathrm{~h}$ (h) AEL over an 18-h period. After fixation, as described above, $\mathrm{MeOH}$ was removed and embryos were transferred into glass dishes with PBST. They were then hand-dissected with Dumont \#5 forceps. For staging, embryos were incubated with 1:1000 SYTOX Green (Invitrogen) in the dark for $30 \mathrm{~min}$ at room temperature. They were then washed three times with PBST and visualized under fluorescence microscopy (Olympus SZX12, Leica 501007, or Leica SP5X). D. melanogaster protocols were followed for tracking the cytoskeletal dynamics using phalloidin and DAPI nuclear staining [42]. For phalloidin staining, $80 \% \mathrm{EtOH}$ was used instead of $\mathrm{MeOH}$ for fixation. After hand-dissection in PBTA (1× PBS, $0.1 \%$ TritonX-100, $0.02 \%$ sodium azide), embryos were incubated with Alexa Fluor 488 phalloidin (1:200; Molecular probes) overnight at $4{ }^{\circ} \mathrm{C}$ and then washed several times with PBST. Embryos were mounted in Vectashield mounting solution with DAPI (Vector Laboratories) and visualized with confocal microscopy (Leica SP5X). For in situ hybridization, digoxygeninlabeled Dmac-prd probes were synthesized using T7 polymerase (antisense) or T3 polymerase (sense) (Roche). The in situ hybridization was performed following modifications of a standard D. melanogaster RNA in situ hybridization protocol [43] (see Additional file 2 for details). Briefly, fixed embryos were hand-dissected in PBST. Embryos were pre-hybridized in hybridization solution for one $\mathrm{h}$ at $60^{\circ} \mathrm{C}$. After overnight incubation with 1:50 of digoxygenin-labeled probe $(\sim 10 \mathrm{ng} / \mu \mathrm{l}$ final concentration) at $60{ }^{\circ} \mathrm{C}$, embryos were washed in hybridization solution and PBST. AP conjugated sheep anti-digoxygenin antibody (1:2000; Roche) was added. Embryos were incubated for one $h$ at room temperature. Following four washes with PBST, NBT/BCIP (Roche) was used for detection. Antibody staining was performed following a standard D. melanogaster protocol $[44,45]$. Hand-dissected fixed embryos were incubated with antiEn 4D9 primary antibody (1:5 dilution of antibody stock provided by the Developmental Studies Hybridoma Bank at $53 \mu \mathrm{g} / \mathrm{ml}$ ) and then with biotinylated anti-mouse antibody (1:500; Vector Laboratories). A color reaction was performed after $\mathrm{ABC}$ (Vector Laboratories) incubation using DAB (Sigma). Embryos were incubated with SYTOX Green in PBST, washed three times in PBST, and visualized with Olympus SZX12, Leica 501007, or Zeiss SteREO Discovery. V12 microscopy. Embryos at germ band stages were hand-dissected to remove yolk before visualization.

\section{Parental and embryonic RNA interference and phenotypic analysis}

Primers with T7 promoter sequence at their $5^{\prime}$ ends were used to amplify fragments from KS-Dmac-prd. $\left(5^{\prime}\right.$ region: STPYAP to VQPSSS, forward primer: DmacprdRNAi5'F: $5^{\prime}$ taatacgactcactatagggagaTTCAACTCCATACGCACCAA, reverse primer: Dmac-prdRNAi5'R: $5^{\prime}$ taatacgactcactatagggagaTGATGAACTCGGTTGCACAT; $3^{\prime}$ region: SANSNS to NPSKTF, forward primer: Dmac-prdRNAi3'F: $5^{\prime}$ taatacgactcactatagggagaAGTGCCAATAGCAACAGCAA, reverse primer: Dmac-prdRNAi3'R: $5^{\prime}$ taatacgactcactatagggagaCCGAAGGTTTTTGATGGATT). The PCR products were used as templates for dsRNA syntheses. MEGAscript T7 Transcription kit (Ambion) was used to make dsRNA according to the manufacturer's instructions. For parental RNAi, pupae were selected from the $D$. maculatus colony. Female and male pupae were separated by visualizing their genitalia (Additional file 3). $2 \mu \mathrm{l}$ of dsRNA $(2 \mu \mathrm{g} / \mu \mathrm{l})$ was injected into the abdomen of each newly eclosed female. After 1 day recovery at $30^{\circ} \mathrm{C}$, injected females were mated by placing them in small plastic cages with an equal number of uninjected males. After allowing them to mate for 1 day, cotton balls were added to cages and embryos were collected daily for phenotypic analysis. For embryonic RNAi, 0-3 h AEL embryos (pre-cellular blastoderm) were collected at $25{ }^{\circ} \mathrm{C}$ and aligned on glass slides. Approximately 50-100 ng $(3 \mu \mathrm{g} / \mu \mathrm{l})$ dsRNA was injected into each embryo using a micromanipulator within $5 \mathrm{~h}$ AEL. To examine morphological defects, hatched larvae were collected and fixed in \#1184C Pampel's solution (BioQuip Products, Inc.) at $4{ }^{\circ} \mathrm{C}$ overnight before visualization. To screen for segmentation defects, each larva was stretched 
out using forceps under a dissecting microscope. To examine Engrailed (En) expression, embryos at appropriate stages were fixed and stained, as described above.

\section{Results}

\section{Early embryogenesis in D. maculatus}

Since little was known about the early stages of $D$. maculatus embryonic development, we tracked nuclear and cytoskeletal dynamics using SYTOX Green, DAPI and phalloidin staining (Fig. 1). Progression of embryogenesis was monitored at $25{ }^{\circ} \mathrm{C}$ to slow development and capture all stages. Zygotic nuclei were first observed dividing multiple times in the center of the embryo, forming a syncitium (0-6 h AEL, Fig. 1a-c). At very early stages, female and male pronuclei were evident inside the embryo (white arrow, Fig. 1a), while the polar body nuclei were at the surface of the embryo (red arrow, Fig. 1a). After several divisions, zygotic nuclei gradually distributed along the length of the embryo (Fig. 1b) and, after additional divisions, began migrating toward the egg surface (Fig. 1c). Between 6 and $8 \mathrm{~h} \mathrm{AEL}$, most of the nuclei had migrated to the periphery of the egg, forming a syncytial blastoderm (Fig. 1d). "Cap"-like phalloidin staining was detected in some embryos at this stage, suggesting that nuclei arriving at the surface of the embryo are surrounded by cytoplasmic regions containing cytoskeleton (Fig. 1e). These phalloidin-stained actin caps protruded at the embryo surface, similar to cytoskeletal events that occur at a comparable stage in D. melanogaster (cell cycle 9/10; [46, 47]). Later, cell membranes formed between individual energids (nucleus with associated cytoplasm) as "furrow canal"-like phalloidin staining appeared, and a cellular blastoderm was established (8-10 h AEL, Fig. $1 \mathrm{~g}$ ). This is similar to cellularization events in $D$. melanogaster at cell cycle $14[46,47]$. In D. maculatus, we were able to capture embryos in which dividing cells with two nuclei still sharing cytoplasm were visible at the cellular blastoderm surface (arrows, Fig. 1h), while cells that had finished cytokinesis each exhibited one nucleus enclosed by its own, individual membrane (Fig. 1i).

Between 10 and $12 \mathrm{~h} \mathrm{AEL}$, the D. maculatus embryo was rapidly transformed from a uniform cellular blastoderm to an elongating germ band (Fig. 1j, k, Additional file 4). In late cellular blastoderm, cells in the ventral posterior region packed together, forming the germ rudiment (Additional file 4a). The first detectable sign of gastrulation was the formation of a ventral furrow (vf), which appeared as a shallow broad furrow in the midventral region (Additional file 4b, b'). Shortly after, several transverse folds emerged (Additional file 4b). As the ventral furrow further invaginated into the interior of the egg, it elongated towards the ventral posterior end (Additional file $\left.4 \mathrm{c}^{\prime}\right)$. The anterior-most fold embedded deeper while other short-lived transverse folds became invisible due to cell movements (Additional file 4c, c'). The dorsal embryonic region condensed while the dorsal anterior extraembryonic region expanded with gastrulation progression (compare Additional file $4 \mathrm{~b}, \mathrm{c}$, arrowheads indicate the boundary between extraembryonic region and the embryo proper). Gastrulation proceeded as the ventral furrow became narrower and reached the posterior end (Fig. 1j; Additional file 4d, d'). Head lobes (hl) were visibly distinguished from surrounding extraembryonic tissue (Fig. 1j; Additional file 4d, d'). During the same time period, a posterior amniotic fold (paf) emerged and, shortly after, covered the posterior end of the germ anlage (red arrow in Fig. 1j; red arrowhead in Additional file 4d). It continued to proceed anteriorly along the ventral side as the germ band elongated (red arrowhead, Additional file $\left.4 \mathrm{e}, \mathrm{e}^{\prime}\right)$. By approximately $12 \mathrm{~h}$ AEL, an early germ band with serosal window (sw) was established (Fig. 1k, red dashed line). The germ band further extended dorsally over the next $4 \mathrm{~h}$ and segmental furrows appeared in an anterior to posterior progression (12-16 h AEL; white arrowheads in Fig. 1l, m). Morphological segments as well as appendage primordia were seen at 16-18 h AEL (red arrowheads in Fig. 1n).

In sum, D. maculatus embryogenesis progressed through pre-blastoderm, cellular blastoderm, gastrulation and germ band extension stages within the first $18 \mathrm{~h}$ AEL at $25{ }^{\circ} \mathrm{C}$. As expected, at $30{ }^{\circ} \mathrm{C}$, embryos developed faster: a cellular blastoderm formed and gastrulation began between 4 and $6 \mathrm{~h} \mathrm{AEL}$. An early germ band was established 6-8 h AEL and the embryo reached late germ band stages within $10 \mathrm{~h}$ AEL (Additional file 5).

\section{Isolation of prd from D. maculatus}

To identify Dmac-prd ortholog(s), degenerate primers were designed based on conserved sequences in the paired domain (PD) and the homeodomain (HD) in Pax3/7 orthologs $[38,39,48,49]$. An approximately $600 \mathrm{bp}$ fragment isolated by PCR amplification using Dmac 0-1 day cDNA was extended by two rounds of 3'RACE to generate a $1341 \mathrm{bp}$ fragment that encodes a PD and a HD (Fig. 2; Dmac-prd GenBank Accession number KT875123). An octapeptide sequence (OP) is present in most Pax3/7 orthologs but is absent from Prd from D. melanogaster, T. castaneum, Apis mellifera (A. mellifera) and Nasonia vitripennis ( $N$. vitripennis) [50, 51]. This OP was not found in the Dmac-Prd sequence, consistent with this being an ortholog of $p r d$, rather than another family member. The HD of this predicted DmacPrd has a serine residue at position 50 (red arrow, Fig. 2), which is vital for the DNA-binding specificity of Prdfamily homeodomains $[26,38,50,52]$. As shown in Fig. 2, the PD and the HD from D. maculatus, T. castaneum and 

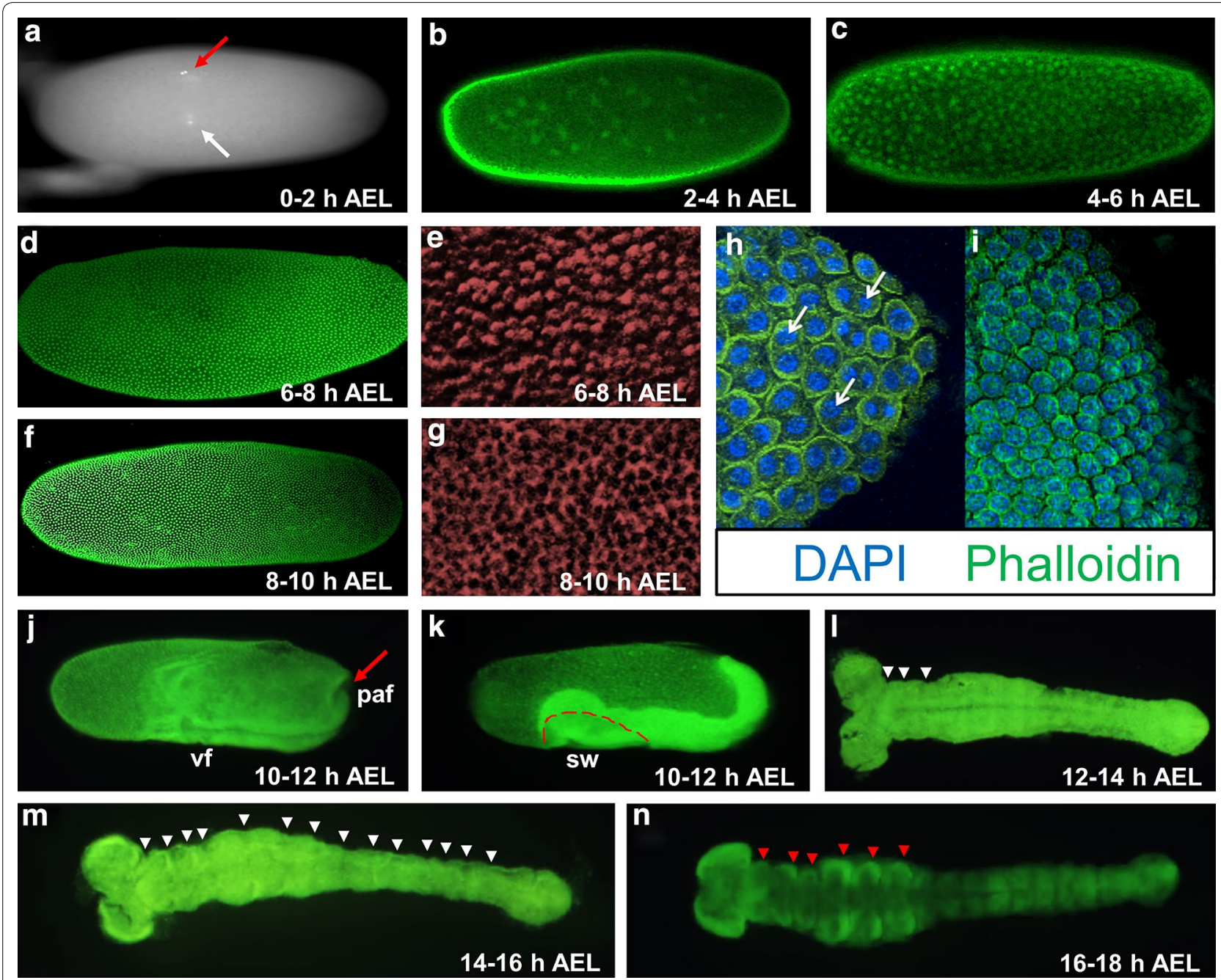

Fig. 1 Early D. maculatus embryogenesis. Photographs of D. maculatus embryos are shown, documenting key steps of nuclear division and early embryonic development. a DAPI nuclear staining of a 0-2 h AEL D. maculatus embryo. b-d, $\mathbf{f}, \mathbf{j}-\mathbf{n}$ Nuclear staining using SYTOX Green of $D$. maculatus embryos between 2 and $18 \mathrm{~h} \mathrm{AEL}$, as indicated. e, $\mathbf{g}$ F-actin phalloidin staining of 6-8 $\mathrm{h}$ and $8-10 \mathrm{~h} \mathrm{AEL} \mathrm{D.} \mathrm{maculatus} \mathrm{embryos} \mathrm{(recolored}$ red). h, i Merge of DAPI (blue) and phalloidin (green). a White arrow indicates pronuclei. Red arrow indicates polar body nuclei. b Nuclei have divided and spread in the central portion of the embryo. c Nuclei continue to divide and migrate towards the egg surface. $\mathbf{d}$ Most nuclei have arrived

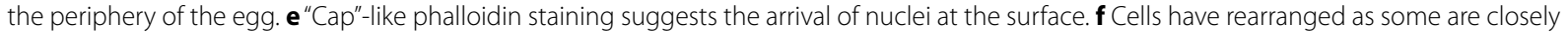
clustered together in the ventral posterior area. $\mathbf{g}$ "Furrow canal"-like phalloidin staining appears during this stage. $\mathbf{h}$, i Fully cellularized embryo. White arrows indicate cells at telophase of mitosis on the egg surface. $\mathbf{j}$ The ventral furrow (vf) has invaginated and posterior amniotic fold (paf, red arrow) has appeared. $\mathbf{k}$ The germ band has coalesced and begun to extend towards the dorsal side of the embryo. Red dashed line indicates serosal window (sw). I An extending germ band stage embryo with bilateral head lobes. White arrowheads show segmental furrows. $\mathbf{m}$ Segmental furrows appear in more posterior regions as the germ band elongates (white arrowheads). $\mathbf{n}$ A fully elongated germ band with morphological segments and appendage primordia (red arrowheads indicate appendage primordia). Embryos were reared at $25^{\circ} \mathrm{C}$ and photographed with Olympus SZX 12 , Leica 501007 or Leica SP5X confocal microscopy

D. melanogaster are similar. The PD of Dmac-Prd is $97 \%$ identical to that of Tc-Prd, with only 3 amino acid differences in the $\mathrm{N}$-terminal portion of the PD, and is $84 \%$ identical to that of Dm-Prd. The Dmac-Prd HD is $98 \%$ identical to that of $T c$-Prd, with only the most C-terminal amino acid different, and $92 \%$ identical to the Dm-Prd HD. Blastx searches using sequences of other TA cloning products identified orthologs of $g s b$ and $g s b-n$ in that their predicted protein sequences possess a PD, a HD and a Gsb- or Gsb-n-type OP ( $g s b$ and $g s b-n$ GenBank Accession number KT875128 and GenBank Accession number KT875129; Additional file 6).

\section{Dmac-prd is expressed in stripes}

To investigate the expression of prd in D. maculatus, RNA in situ hybridization in early embryos was 


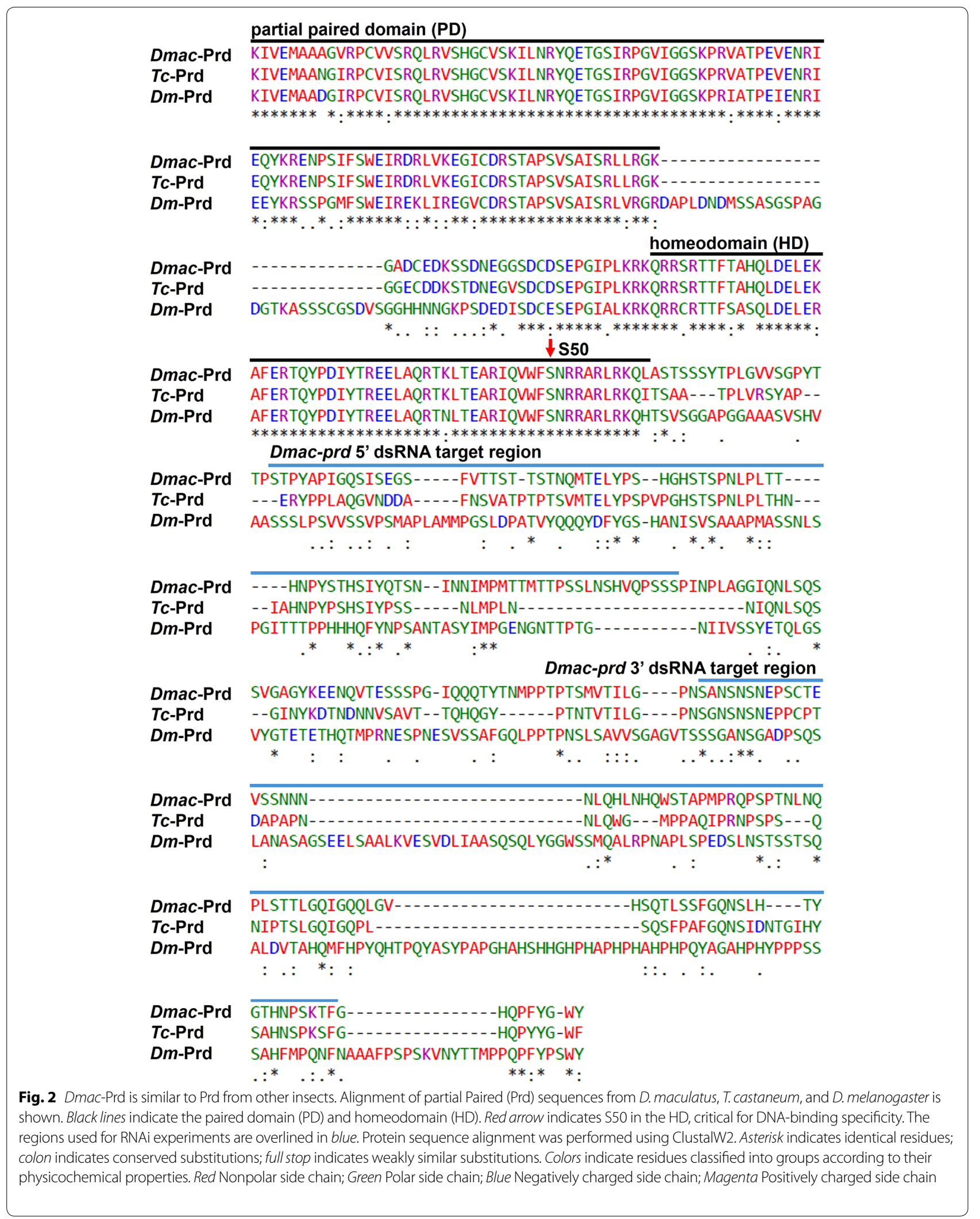


performed. No specific staining pattern was detectable using a sense probe (data not shown). Using an antisense probe, Dmac-prd transcripts were initially detected as a single stripe at approximately $50 \%$ of the blastoderm length (black arrow, Fig. 3a). Posterior Dmac-prd stripes emerged sequentially in an anterior to posterior fashion (Fig. 3b-n). The first primary Dmac-prd stripe resolved into a more clearly detectable thin stripe and remained undivided (black arrow, Fig. 3b, c). The second and the third primary Dmac-prd stripes first appeared as weak broad stripes in the posterior half of the embryo (red arrows, Fig. 3b, c). These two primary stripes split into pairs of thin secondary stripes (red arrowheads, Fig. 3d, e). By the time the fourth primary Dmac-prd stripe arose in the posterior region (late cellular blastoderm, red arrow, Fig. 3d), the second primary stripe had completed its split into two secondary stripes (red arrowheads, Fig. 3d), and the third primary stripe began to split (black arrowhead, Fig. 3d). At the onset of gastrulation when the ventral furrow emerged, the anterior-most undivided Dmac-prd stripe, four anterior secondary stripes and a fourth primary stripe were clearly observed (Fig. 3e).

During gastrulation, when the ventral furrow had invaginated further into the yolk and several transverse folds appeared, the fourth primary stripe had resolved into secondary stripes (arrowheads, Fig. 3f, g) and a fifth primary stripe was detected (red arrow, Fig. 3g). When the posterior invagination and the ventral furrow became more prominent (black arrowhead), a total of eight prd stripes (5 primary stripes, among which the first remained undivided, three middle stripes split into 6 secondary stripes, and a fifth newly arisen stripe, red arrow) were detected (Fig. 3h). Anterior stripes started to fade while posterior stripes were embedded into the posterior end due to the SAZ invagination (Fig. 3i, j). As gastrulation proceeded, the embryonic rudiment with bilateral head lobes was clearly distinguishable from the extraembryonic tissue (Fig. 3i, j, red dashed line in i indicates the anterior boundary of the germ rudiment). In the embryonic rudiment, secondary stripes resolved from the fifth primary stripe (red arrowheads, Fig. 3i, j) and a weak sixth primary stripe (red arrow, Fig. $3 i$, j) was detected.

As the germ band extended, new prd stripes arose from the region anterior of the SAZ and resolved into thin secondary stripes by fading expression in the center (Fig. $3 k-n$ and $3 p-r)$, as reported in other species [26, 53-55]. There was no obvious intensity or width difference within pairs of Dmac-prd secondary stripes in the blastoderm or the germ band (Fig. 3e-g and p-r). As posterior Dmac-prd stripes were added sequentially, anterior prd stripes became weak and eventually invisible (Fig. 3k-n). Gnathal, thoracic and abdominal prd stripes disappeared gradually during germ band extension
(Fig. 3l-n). During later embryogenesis, prd was strongly expressed in appendage primordia in gnathal segments (black arrows, Fig. 3o). Together, the conserved protein sequence, expression in stripes, and the characteristic splitting of primary stripes into secondary stripes in early embryos, suggested that Dmac-prd is involved in pairrule patterning. The finding that a total of four primary Dmac-prd stripes are present at the onset of gastrulation is consistent with the assignment of $D$. maculatus as an intermediate-germ insect.

\section{RNAi knockdown of Dmac-prd results in defects in segmentation}

To investigate the function of $p r d$ in D. maculatus, and to determine whether RNA interference (RNAi) is effective in this species, we performed embryonic RNAi (eRNAi). Dmac-prd 3' dsRNA, corresponding to a 254 bp region downstream of the HD, was injected into pre-blastoderm stage embryos (target region is indicated in Fig. 2). After injection, all hatched offspring from control embryos injected with $g f p$ dsRNA were wild type in appearance with head, three thoracic segments and ten abdominal segments (Fig. 4a). In contrast, over $85 \%(18 / 21)$ of the newly hatched larvae after Dmac-prd $3^{\prime}$ dsRNA injections showed segmentation defects with one or several fused segmental boundaries (T2/T3, A1/A2, A3/A4, A5/A6, A7/A8; black arrows in Fig. $4 \mathrm{~b}-\mathrm{d}$ ), reminiscent of the segmentation phenotype produced by eve eRNAi in cricket [56]. Some cases included loss of or abnormal development of T2 legs (red arrow, Fig. 4d).

Injection of dsRNA into pupal or adult females can result in phenotypes evident in their offspring. This phenomenon was named parental RNAi (pRNAi) and has been observed in T. castanum, O. fasciatus, Gryllus bimaculatus, Blattella germanica, N. vitripennis, and other species [57-61]. To determine whether pRNAi functions in D. maculatus, and to verify the segmentation phenotypes observed with Dmac-prd eRNAi, Dmac-prd $3^{\prime}$ dsRNA was injected into newly eclosed virgin females and their offspring were examined. To ensure specificity, a second dsRNA was generated from a non-overlapping target region (Dmac-prd 5', 256 bp; Fig. 2). There was no significant difference in the offspring yield or hatch rates between $g f p$ dsRNA injected and Dmac-prd $5^{\prime}$ or $3^{\prime}$ dsRNA injected females (data not shown). Segmentation in all hatched offspring from control females injected with $g f p$ dsRNA appeared to be wild type (Fig. 4e). In contrast, over $50 \%(100 / 184)$ of hatched offspring collected on the 3rd day after injection from Dmac-prd 3' dsRNA injected females and $73 \%$ (66/91) from Dmacprd $5^{\prime}$ dsRNA injected females displayed segmentation defects (Figs. 4f-h, 5a). The percentage dropped to 
a

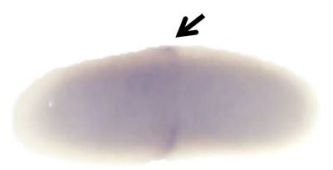

b

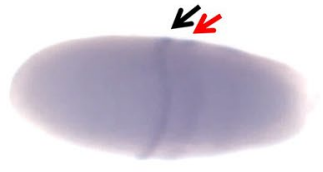

C

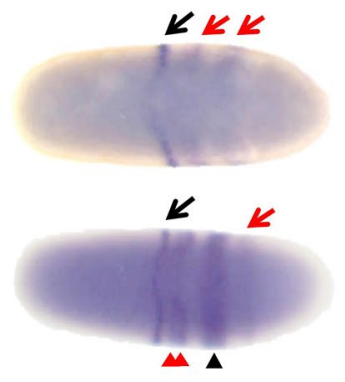

e

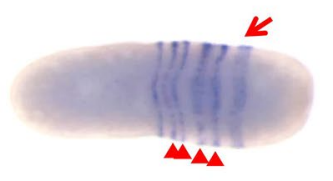

$\mathbf{f}$

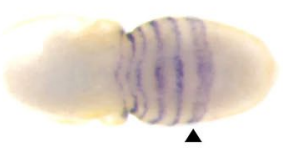

g

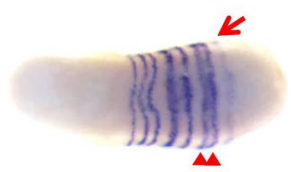

h

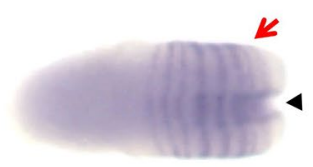

i

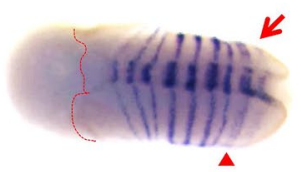

j

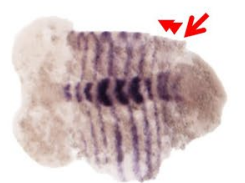

k

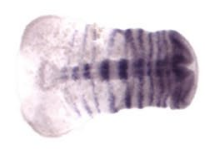

I

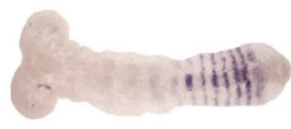

m

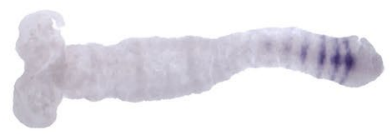

n

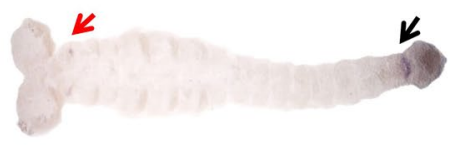

0

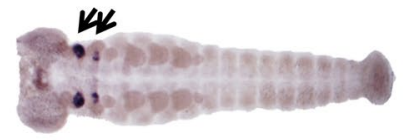

p

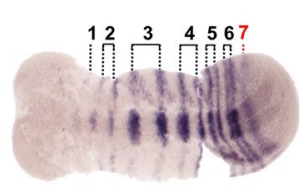

$q$

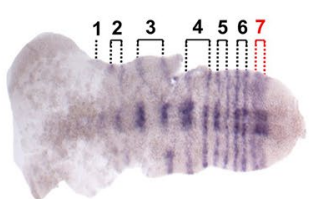

r

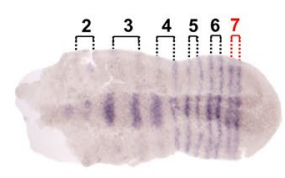

Fig. 3 Dmac-prd is expressed in stripes during embryogenesis. Expression of Dmac-prd examined by in situ hybridization. Arrows and arrowheads indicate primary and secondary stripes, respectively. Black arrows show "old" primary stripes while red arrows indicate "new" primary stripes. Black arrowheads show splitting primary stripes, and red arrowheads indicate resolved secondary stripes. a A single weak stripe in early blastoderm (black arrow). b The first stripe becomes clearly detectable (black arrow). The second stripe emerges posterior to the first stripe (red arrow). c Two broad primary stripes appear (red arrows). d A late blastoderm stage embryo. The first primary stripe remains undivided (black arrow). The second primary stripe has divided into two secondary stripes (red arrowheads). The third primary stripe is splitting (black arrowhead). The fourth primary stripe is showing up de novo (red arrow). e When the broad shallow ventral furrow appears, the first undivided stripe, four secondary stripes (red arrowheads) and a fourth primary stripe are detected (red arrow). f Fading expression is detected in the center of the newly arisen stripe (black arrowhead). $\mathbf{g}$ The fourth primary stripe has divided into two stripes (red arrowheads). A weak fifth stripe appears (red arrow). h During gastrulation, a total of 8 Dmacprd stripes are detectable. Black arrowhead indicates the posterior end of the ventral furrow. Red arrow indicates the posterior-most Dmac-prd stripe. $\mathbf{i}, \mathbf{j}$ As gastrulation proceeds, a 6th primary stripe arises; bilateral head lobes become visible. Red arrowheads indicate the dividing stripe. Red arrow indicates the newly emerged stripe. Red dashed line in $\mathbf{i}$ shows the anterior edge of the germ rudiment. $\mathbf{k}$ Embryo during early germ band elongation with striped Dmac-prd expression across the whole germ band. I, $\mathbf{m}$ Elongating embryo with faint Dmac-prd stripes in anterior segments. Posterior segments have strong striped Dmac-prd expression. $\mathbf{n}$ Embryo at late germ band elongation stage. Stripes have faded except for the most posterior segment (black arrow). Hint of Dmac-prd expression appears in the mandibles (red arrow). $\mathbf{o}$ Later embryo showing Dmac-prd expression in the head (black arrows). p-r Detailed view of stripe splitting. p A total of 7 primary stripes have developed. The first stripe remains undivided. The next 5 primary stripes have resolved to secondary stripes. The 7th primary stripe emerges from the anterior region of the posterior end of the embryo as a broad weak stripe. q Anterior striped expression fades. The expression in the center of the 7th stripe becomes fuzzy and faint. $\mathbf{r}$ The 7th stripe has divided into two thin secondary stripes as there is no expression in the center. All embryos are shown with anterior to the left

less than $30 \%(51 / 195)$ and $39 \%(52 / 135)$ on the 4 th day after injection for Dmac-prd $3^{\prime}$ and Dmac-prd $5^{\prime}$, respectively (Fig. $5 \mathrm{a}$ ). On the 5 th day after injection, less than $3 \%$ of embryos hatched with segmentation defects (Dmac-prd 5', 6/202; Dmac-prd 3' 9/305; Fig. 5a). Only very few embryos collected on the 6th day after injection 
a

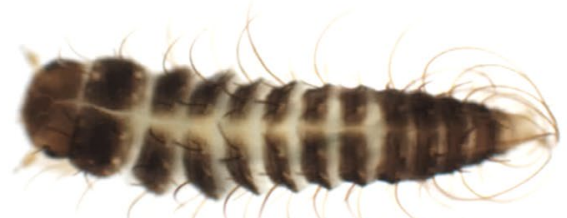

gfp eRNAi

C

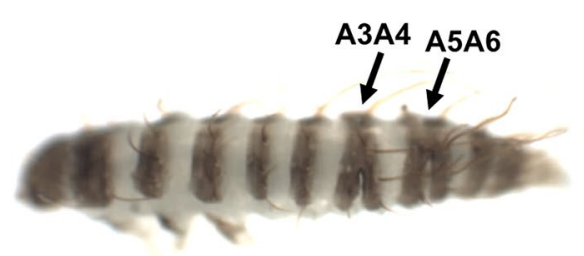

prd eRNAi

e
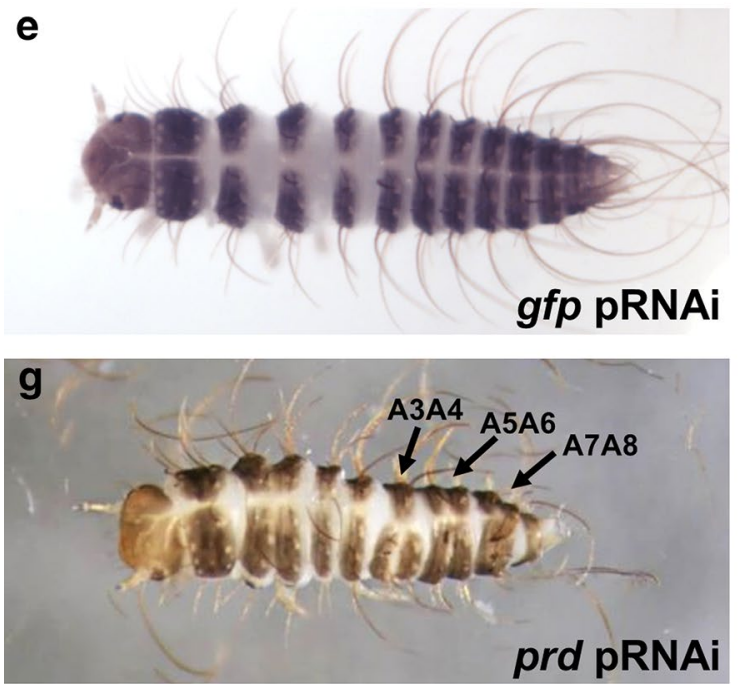

b

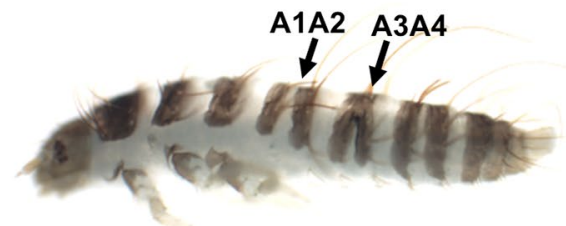

prd eRNAi

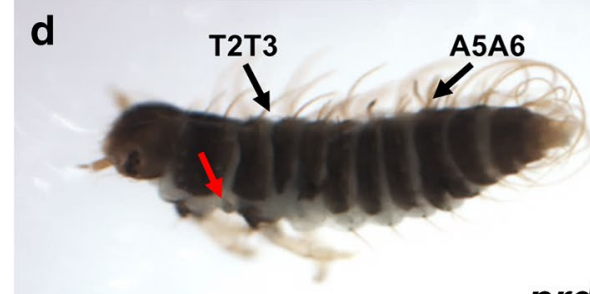

prd eRNAi
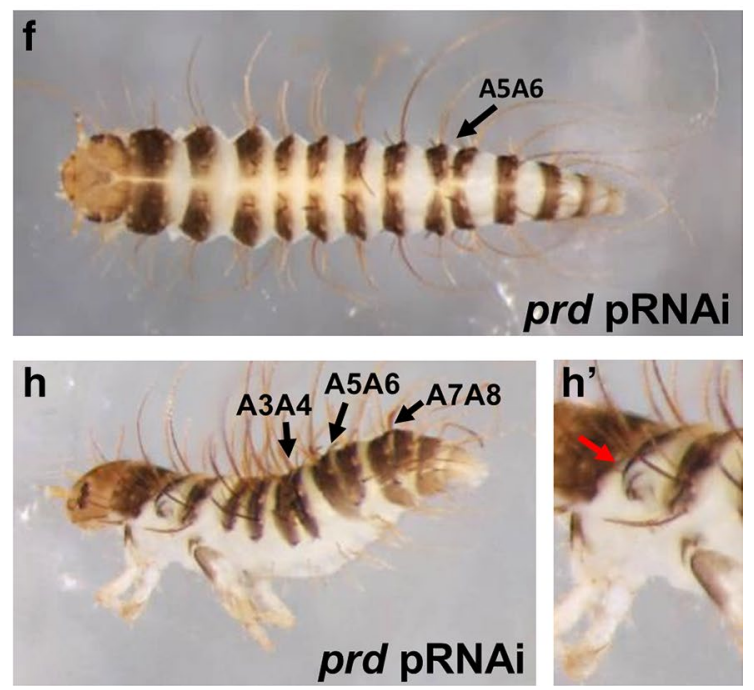

Fig. 4 Knockdown of Dmac-prd with RNAi causes segmentation defects. Dmac-prd or gfp RNAi was carried out, as indicated. gfp dsRNA was injected as negative control. a-d Embryonic RNAi. e-h Parental RNAi. a Dorsal view of a first instar D. maculatus larva after gfp dsRNA injection showing wild-type phenotype with head, three thoracic segments and ten abdominal segments. b A hatched first instar larva after Dmac-prd dsRNA injection contains fused A1/A2 and A3/A4 segments (black arrows). c Lateral view of a larva with fused A3/A4 and A5/A6 segments after Dmac-prd eRNAi (black arrows). d T2 legs are missing in hatched larva with severe phenotype after Dmac-prd eRNAi (red arrow). Black arrows indicate fused T2/T3 and A5/A6 segments. e Offspring produced by gfp dsRNA injected female are viable until hatching and show wild-type phenotype (dorsal view). f Dorsal view of a hatched offspring with fused A5/A6 segments from Dmac-prd $3^{\prime}$ dsRNA injected female (black arrow). g, h First instar larva after Dmac-prd ( $3^{\prime}$ and $5^{\prime}$, respectively) pRNAi with shortened body length as well as fused segments. Black arrows indicate fusions of adjacent segments. h' Red arrow indicates defective T2 leg

hatched with fused segments $\left(2 / 234\right.$, Dmac-prd $5^{\prime} ; 1 / 282$, Dmac-prd 3'; Fig. 5a).

Analysis of segmentation defects revealed a range of defects, phenocopying an allelic series. In mildly affected larvae, partial or complete fusion was observed for one pair of adjacent segments, most often A5/A6 (Figs. 4f, $5 \mathrm{~b})$. In other cases, fusions were detected between two, three or four adjacent segments (Figs. 4g, h, 5c). The fusions occurred in the same alternating fashion as observed for eRNAi (Figs. 4f-h, 5b). Missing or defective T2 legs were also observed in some severe cases. Very often, the defective T2 legs projected from the lateral edge of instead of the ventral-lateral side of the T2 segment (red arrow, Fig. 4h').

To further analyze the role of Dmac-prd in segmentation, defects were quantitated in hatched embryos 

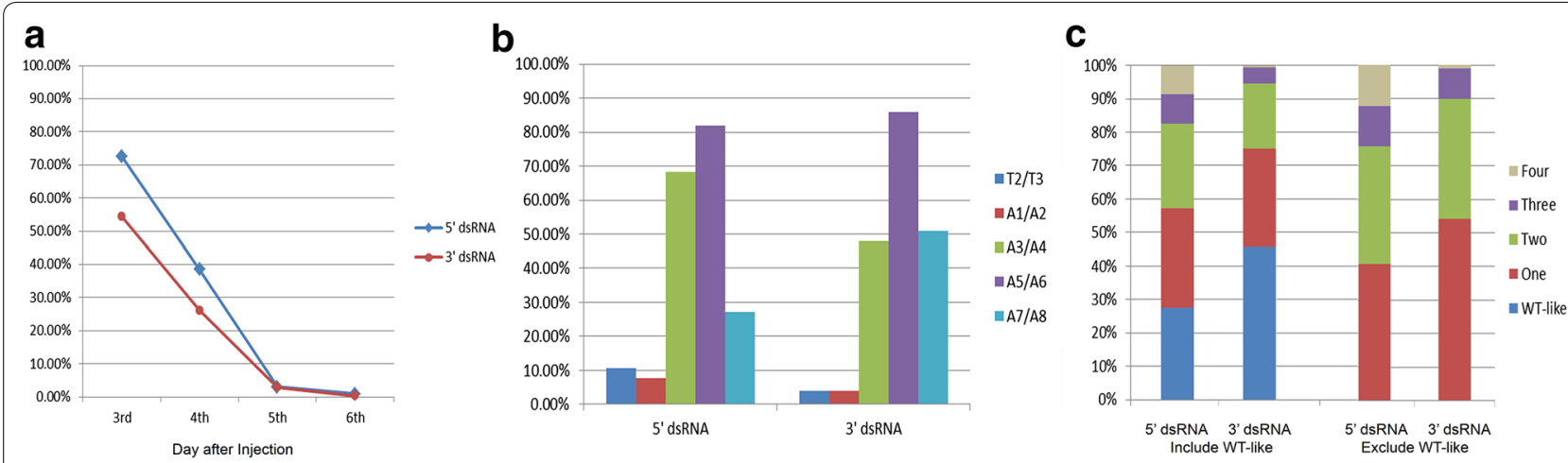

Fig. 5 Quantitation of Dmac-prd pRNAi segmentation defects. Offspring produced by twelve of either Dmac-prd $5^{\prime}$ or $3^{\prime}$ dsRNA injected females, as indicated. Each hatched larva was stretched out using tweezers and examined under a dissecting microscope. a Percent of hatched pRNAi offspring showing segmentation phenotypes. b-c Embryos were collected on the third day after injection and segmentation defects were scored. $\mathbf{b}$ Frequency of fusion of specific pairs of adjacent segments. Note that some larvae had more than one pair fused. $\mathbf{c}$ Frequency of types of segmentation defects observed. Left bars percentage of hatched larvae with wild-type segmentation, and those displaying one, two, three or four segmental fusions; right bars percentage of hatched larvae with one, two, three or four segmental fusions among those with observable segmentation defects

collected on the third day after injection. More than $70 \%$ of Dmac-prd 5' dsRNA offspring displayed some type of defect (Fig. 5a). Of these, $\sim 40 \%$ displayed one segmental fusion while nearly $60 \%$ hatched with more than one segment fused (35\% with two fusions, $12 \%$ with three fusions and $12 \%$ with four fusions; Fig. $5 \mathrm{c}$ ). The percentage of Dmac-prd $3^{\prime}$ dsRNA affected offspring was over $50 \%$ (Fig. $5 \mathrm{a}$ ). Of these, $54 \%$ had one segmental fusion, $36 \%$ had two, $9 \%$ had three, and $1 \%$ had four segments fused (Fig. 5c). Overall, segments A5/A6 were most commonly affected by Dmac-prd knockdown, with over $80 \%$ of either Dmac-prd $5^{\prime}$ or $3^{\prime}$ dsRNA affected larvae displaying fusion of these segments (Fig. 5b). Fusion of A3/A4 was seen in $68 \%$ and $48 \%$ of Dmacprd $5^{\prime}$ and $3^{\prime}$ affected larvae, respectively. Fusion of A7/ A8 was detected in 27 and $51 \%$ of Dmac-prd $5^{\prime}$ and $3^{\prime}$ affected larvae, respectively. Fusions of T2/T3 and A1/ A2 had lower frequencies (11 and $4 \%$ for fused T2/T3 in Dmac-prd $5^{\prime}$ and $3^{\prime}$ affected larvae, respectively; 8 and $4 \%$ for fused A1/A2 in Dmac-prd $5^{\prime}$ and $3^{\prime}$ affected larvae, respectively). These differences in frequency suggest differential susceptibility of different parasegments to Dmac-prd knockdown (Fig. 5b).

In sum, both eRNAi and pRNAi were effective tools to analyze gene function in $D$. maculatus. Analysis of the morphology of larvae hatched after knockdown of Dmac-prd indicates a role for prd in segmentation in this species.

\section{Dmac-prd is necessary for the expression of alternate Engrailed stripes}

In both $D$. melanogaster and T. castaneum, prd functions as a pair-rule gene and regulates en expression in odd-numbered segments [26, 62]. We therefore asked if Dmac-prd functions similarly to regulate the expression of alternate En stripes in D. maculatus. Embryos injected with buffer alone showed equally strong En expression in every segment (Fig. 6a). In contrast, loss of En expression in alternating segments was evident in over $50 \%$ (25/46) of extended germ bands after Dmac-prd eRNAi (asterisks, Fig. 6c, e). Germ band morphology was also analyzed using nuclear staining with SYTOX Green. This revealed partial or even complete fusion of pairs of adjacent segments into a wider segment (asterisks, Fig. 6d, f).

Since injection of embryos may have caused damage that precluded a more careful analysis of En expression, embryos laid by Dmac-prd dsRNA-injected females were also examined. While offspring from the $g f p$ dsRNA control injected females displayed wild-type-like En expression (Fig. 6g), loss of or reduced En expression in the labium, T2, A1, A3, A5, A7 and A9 segments were detected in over $60 \%(112 / 179)$ of extended germ band stage embryos from Dmac-prd dsRNA injected females (asterisks, Fig. 6i). Segmental fusion was observed in the posterior region of odd-numbered segments following nuclear staining in the regions where loss of En expression was detected (asterisks, Fig. 6j).

The decreased expression of alternate En stripes, as well as the segmentation defects observed in embryos in which Dmac-prd was knocked down, indicate that Dmac-prd functions as a pair-rule segmentation gene in D. maculatus.

\section{Discussion}

Here we have established $D$. maculatus as a new system for studying embryonic development, gene expression 

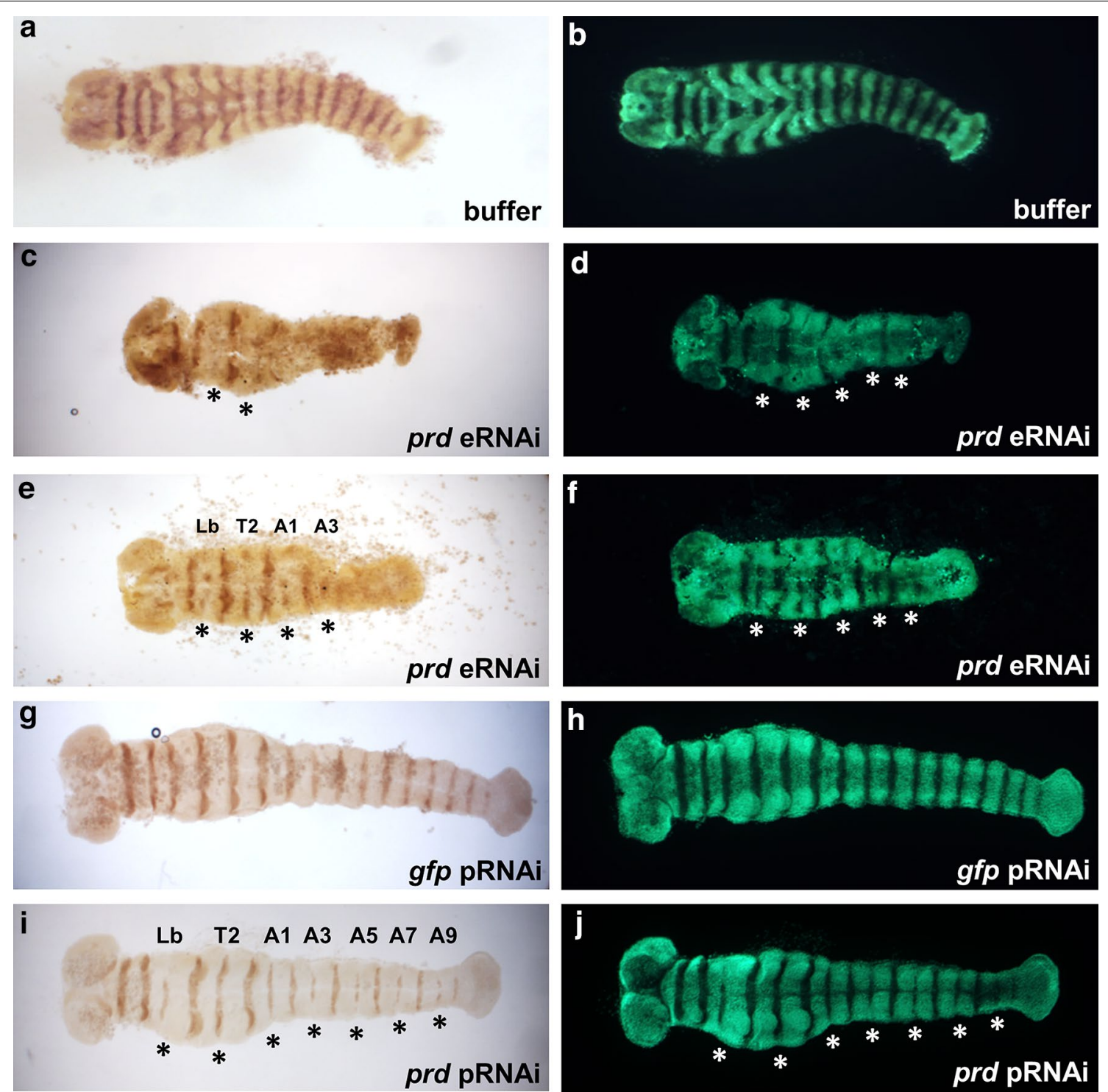

Fig. 6 Reduced expression of alternate Engrailed stripes after Dmac-prd RNAi. a-f Embryonic RNAi. $\mathbf{g}-\mathbf{j}$ parental RNAi. a, c, e, g, i Injected embryos 24-27 h AEL (eRNAi) or 0-1 d AEL embryos from injected females (pRNAi), as indicated were fixed and stained using anti-En 4D9 primary antibody and DAB staining. $\mathbf{b}, \mathbf{d}, \mathbf{f}, \mathbf{h}, \mathbf{j}$ SYTOX Green nuclear staining of same embryos for visualization of morphological defects. Asterisks indicate reduced En expression, fused segments or partial fusion between two neighboring segments

and gene function. D. maculatus were maintained in long-term culture in the lab and large numbers of embryos were readily collected and processed. The timing and progression of nuclear divisions, cellularization, gastrulation, and germ band development were described (Fig. 1; Additional file 4). Genes of the Pax3/7 family were isolated (Fig. 2; Additional file 6) and the Dmac-prd ortholog was found to be expressed in stripes in blastoderm, gastrulation and germ band extension stages embryos, with additional stripes added from the posterior region (Fig. 3). Both eRNAi and pRNAi were effective in this species, revealing a role for Dmac-prd in pair-rule patterning (Figs. 4, 5, 6), similar to that seen in other insects [25, 26, 53, 54, 63, 64]. These findings suggest that the role of prd in pair-rule patterning is shared among holometabolous insects with different modes of embryonic development.

Our studies support the classification of D. maculatus as an intermediate-germ beetle, as four primary prd stripes were established in late blastoderm (Fig. 3d). 
In contrast, only one Prd/prd stripe was seen in T. castaneum embryos prior to gastrulation [26, 38]. In $T$. castaneum, the pair-rule segmentation genes hairy and even-skipped (eve) are expressed in two stripes before gastrulation [17, 23, 26, 32, 65]. One En and one wingless stripe were detected at the same stage [17, 32, 66, 67]. In a long-germ beetle, Callosobruchus maculatus, six eve primary stripes were evident before gastrulation [32], while four eve primary stripes were present in late blastoderm Dermestes frischi embryos [32], similar to what we observed for $p r d$.

To our knowledge, this is the first demonstration of RNAi function in dermestids. Dermestid beetles include 500-700 species worldwide. D. maculatus (common name hide or skin beetle), has been widely used for skeletonizing dead animals [68]. It is a worldwide pest for the stored meat industry and also the silk industry because it slaughters silkworm cocoons $[69,70]$. Various dermestid species feed on stored meat, stored grain, silk, cheese, poultry, natural or synthetic fiber and pollen [69]. Because of their large numbers and their ability to occupy such diverse habitats, different beetle species have become economically significant pests for agriculture, forests, fabric, and stored food supplies, thus impacting both households and industry [7, 71]. The use of RNAi as a highly specific and safe method to control insect pests shows promise in a number of different taxa [72]. Our studies suggest that RNAi will be a viable strategy for control of dermestid pests.

\section{Protein motifs mix and match in Pax family members}

prd was the founding member of the metazoan Pax family of transcription factors, part of the genetic toolkit directing animal development [39, 48, 73]. Pax family members have taken on diverse roles in embryonic development, organogenesis and have been implicated in a number of human cancers [51, 74-76]. Pax family proteins are characterized by the presence of multiple protein domains, including a paired domain (PD) composed of a bipartite DNA-binding domain (PAI and RED domains separated by a linker region), an octapeptide (OP), and a paired-type homeodomain (PTHD) [77]. Members of different Pax subfamilies contain different combinations of these protein domains, or even truncated versions of individual domains, imparting diversity in both structure and function to this gene family [39, 40, 48, 78-82]. For example, D. melanogaster Pox-meso and Pox-neuro have the PD but lack a HD [78]. Phylogentic analyses suggest that Pax genes fall into distinct subfamilies, with $p r d$ a member of the Pax3/7 group [81, 82]. Pax3/7 family members generally contain a PD, OP and HD and are represented by both prd and the closely related $g s b$ and $g s b-n$ genes in D. melanogaster, with only prd involved in pair-rule segmentation in D. melanogaster [39-41, 48, 49, 53, 63, 83]. Although both the $\mathrm{PD}$ and HD are shared by all these genes, the OP is present in Gsb and Gsb-n but not in Prd [40, 48]. Similarly, in $T$. castaneum and A. mellifera, the OP motif is present in Gsb and Gsb-n but not in Prd and also is not found in the only $N$. vitripennis Pax3/7 family member $[26,50]$. However, the OP is found in many other Pax proteins: e.g., insect Gsb/Gsb-n, Shaven, Pox-meso and Pox-neuro [50] and mammalian Pax 1/9 and Pax 2/5/8 [84]. Phylogenetic analysis suggests that the OP was a feature of ancestral Pax proteins. The presence of the OP in Gsb and Gsb-n but not in Prd of extant insects suggests that during Pax3/7 evolution, the OP was lost in an ancestral Prd ortholog. Therefore, the absence of the OP serves as a signature motif for identification of prd orthologs $[50,51]$. In this study, of the three prd family member genes isolated, only one lacked the OP (Fig. 2; Additional file 6). Expression and functional results demonstrated it to be a bona fide prd ortholog (Figs. 3, 4, 5, 6), consistent with the utility of using the OP motif as a signature to distinguish among $p r d$ family members.

\section{Pax3/7 function in panarthropods}

Pax3/7 family members have been isolated from a broad range of arthropod groups and from the outgroups, Onychophora and Tardigrada. Expression studies suggest a conserved role in segmentation with segmentally expressed stripes seen for $\mathrm{Pax} 3 / 7$ genes from crustaceans, chelicates, myriapods, two onychophorans and a tardigrade, suggesting that the ancestral function in segmentation was of the segment polarity type, affecting every segment [13-16, 85-87]. Indications of a pair-rule type expression are seen in the millipede, Glomeris marginata, where the Pax3/7 family gene pairberry1 (pby-1) is expressed in stripes in the head and anterior thorax. Although these stripes arise almost simultaneously, their intensity alternates in every other segment $[13,88]$. In the two-spotted spider mite (Chelicerata: Tetranychus urticae), the delayed appearance of alternating stripes of a Pax3/7 is reminiscent of pair-rule-type expression [86, 89]. However, it is only in Pancrustacea, or possibly hexapods, that a clear PR-like expression pattern of Pax3/7 genes is observed [38]. The Schistocerca americana ortholog $p b y-1$ is expressed in a pair-rule-like pattern before it is expressed segmentally [38]. Although a role for Pax3/7 in PR patterning may thus have arisen before the origin of holometabolous insects, it is in this clade that PR expression and function has been most extensively documented.

A detailed comparison of the expression of Dmac-prd to that seen for prd in other holometabolous insects shows similarities and differences within this large clade. 
Dmac-prd expression is initiated as a single stripe in the blastoderm (Fig. 3a). In T. castaneum, prd expression also begins as a single stripe in the presumptive mandibular segment [26]. Unlike prd in these two beetles, $D$. melanogaster Prd first is expressed in a broad anterior region that then resolves into a broad stripe [53]. Posterior Dmac-prd stripes appear sequentially in an anterior to posterior fashion in the blastoderm embryo to generate a total of 4 primary stripes before gastrulation (Fig. 3d). Sequential addition of prd stripes in the blastoderm was also detected in A. mellifera and $N$. vitripennis $[50,55]$. This anterior to posterior progression of stripe formation in the blastoderm was also reported for other pair-rule genes in $T$. castaneum, $N$. vitripennis $a$ and O. fasciatus $[22,23,29]$. In contrast to this, in D. melanogaster, the primary Prd stripes 4 and 7 are expressed earlier than stripes 3, 5, 6 and 8 [53]. Thus, even though Prd stripes do not appear simultaneously in long-germ D. melanogaster, they do not arise sequentially from the posterior end.

The remaining primary Dmac-prd stripes are added from the posterior region during germ band elongation (Fig. 3), as in T. castaneum [26, 38]. As in other species, including $D$. melanogaster, the primary prd stripes in $D$. maculatus split into two secondary stripes (Fig. 3e-g, $\mathrm{p}-\mathrm{r}$ ). As seen in A. mellifera, we did not detect any difference in the intensity or width within pairs of stripes, although differences were reported for $T$. castaneum, $N$. vitripennis and D. melanogaster [26, 38, 50, 53-55]. Therefore, to date, there is no obvious correlation between this feature and germ band mode. During germ band elongation, anterior Dmac-prd stripes fade while stripes in posterior abdominal segments display strong expression (Fig. 3k-n). This feature is shared in T. castaneum and $A$. mellifera $[26,55]$, but equally expressed segmental prd stripes without fading of anterior stripes were observed in late blastoderm and fully elongated $N$. vitripennis and $D$. melanogaster germ band embryos [50, 53]. Since $A$. mellifera, $N$. vitripennis and $D$. melanogaster exhibit a long-germ mode of segmentation, while T. castaneum and D. maculatus show short- and intermediategerm modes, such fading of anterior prd stripes during later embryogenesis cannot be correlated with germ band mode. Later during development, Dmac-prd is strongly expressed in gnathal segments (Fig. 3o). This late prd expression pattern appears to be a common feature in insects examined so far, suggesting a conserved function for prd in head development [50, 53-55, 90, 91]. In sum, although there is some divergence suggesting subtle modulation of prd expression, the early striped expression, the splitting of primary prd stripes, and the late head expression appear to be shared throughout insect taxa.

\section{Dmac-prd functions as a pair-rule gene}

As seen in other RNAi knockdown experiments, both Dmac-prd pRNAi and eRNAi resulted in a graded series of defects. Two non-overlapping target regions were used to perform pRNAi and both gave similar results, suggesting that effects were specific. In pRNAi experiments, the penetrance dropped rapidly within one-week of injection (Fig. 5a). pRNAi in T. castaneum displayed relatively high penetrance after weeks [58]. Whether this difference is specific to Dmac-prd or a general feature of RNAi in $D$. maculatus remains to be determined.

Both eRNAi and pRNAi produced defective larvae with fused segmental boundary/boundaries between T2/ T3 (parasegment 5, ps5), A1/A2 (ps7), A3/A4 (ps9), A5/ A6 (ps11), A7/A8 (ps13). In this graded series, larvae displayed segmentation defects with different levels of severity (Figs. 4, 5c). One parasegment (A5/A6) was more sensitive to RNAi, even with low levels of knockdown (Fig. 5b), as has also been reported in other species for pair-rule mutation or knockdown [29, 92]. En expression was reduced or completely lost in odd-numbered segments in $\sim 50 \%$ of Dmac-prd dsRNA injected embryos and $\sim 60 \%$ of pRNAi offspring (Fig. 6). Together, these findings suggest that Dmac-prd functions as a pair-rule segmentation gene in odd-numbered parasegments by activating en expression. This function is shared with shorter-germ $T$. castaneum and long-germ D. melanogaster $[25,26,62,64]$, and thus appears to be conserved, irrespective of the mode of segmentation.

\section{Conclusions}

Here we have established basic approaches necessary to use D. maculatus as a new insect model system. Methods are available not only for basic research approaches but also for developing alternative and safe methods for control of dermestid pests. D. maculatus represents the diverse clade of Coleoptera and displays an intermediategerm mode of segment addition, making it a good system for comparative studies with shorter-germ T. castaneum and long-germ $D$. malanogaster. These comparative studies were initiated here by the isolation and characterization of the D. maculatus ortholog of prd. Consistent with the role of prd in D. melanogaster and T. castaneum, prd functions as a pair-rule segmentation gene in $D$. maculatus. Thus, prd appears to be a 'core' pair-rule gene that retains pair-rule function in a range of insects that display variation in the function of other pair-rule genes and in the mode of segment addition. 


\section{Additional files}

Additional file 1. COI identification of laboratory reared species. The CO gene from our lab D. maculatus colony was compared to the published D. maculatus COI sequence (GenBank ID HM909035.1). Red arrow shows mismatch. Alignment was performed using ClustalW2.

Additional file 2. Protocols for D. maculatus embryo fixation and whole mount in situ hybridization.

Additional file 3. Female and male D. maculatus pupae. Morphology used to distinguish female and male D. maculatus is shown in this photograph. (A) Two genital papillae at the posterior end of a female pupa (white arrows). (B) Male pupa has a median sternal lobe on the ventral side of the posterior abdomen (black arrow).

Additional file 4. Gastrulation in D. maculatus embryos. Embryos were stained with SYTOX Green. (A) Embryo from overnight collection. Note that nuclei are closely packed together posteriorly with large and loosely arranged nuclei in the anterior dorsal region. (B-E) embryos were collected between 10 and $12 \mathrm{~h} \mathrm{AEL}$ at $25^{\circ} \mathrm{C}$. Left column, lateral view; right column, ventral view of same embryo. ( $\left.B, B^{\prime}\right)$ The ventral furrow $(v f)$ and several transverse folds appear as signs of early gastrulation. White arrowhead indicates the boundary between the embryo proper and extraembryonic tissue on the dorsal side. (C, C') Ventral furrow invaginates towards the yolk. The anterior fold separates the head lobes from the anterior extraembryonic tissue. The boundary between the embryo proper and extraembryonic tissue is indicated by the white arrowhead. $\left(D_{1}, D^{\prime}\right)$ The narrower and deeper ventral furrow reaches the posterior end. The amnion folds over the posterior end of the germ rudiment, forming the posterior amniotic fold (paf). Involuting head lobes (hl) are visible. Red arrowhead shows the edge of the paf. $\left(E, E^{\prime}\right)$ The amnion, together with the serosa, moves anteriorly on the ventral side of the embryo, leaving an open serosal window (sw). Red arrowhead indicates the posterior edge of sw.

Additional file 5. D. mauclatus early embryogenesis at 25 and $30^{\circ} \mathrm{C}$. Embryos were collected every $2 \mathrm{~h} \mathrm{AEL}$ at 25 or $30^{\circ} \mathrm{C}$ over an 18 -h or a 10-h period, respectively. D. maculatus embryogenesis was examined using nuclear and phalloidin staining. Embryos at the end of 8-10 h AEL at $30^{\circ} \mathrm{C}$ are roughly equivalent to $14-16 \mathrm{~h} \mathrm{AEL}$ embryos at $25^{\circ} \mathrm{C}$.

Additional file 6. Alignment of partial Dmac-Gsb, Gsb-n, and Prd protein sequences. Black lines indicate the paired domain (PD), octapeptide (OP) and homeodomain (HD). Note that Dmac-Prd is lacking the OP motif. Gsb has a Gsb-type OP: HSIDGILG. Gsb-n has a Gsb-n type OP:YTIDGILG. Protein sequence alignment was performed using ClustalW2. ${ }^{*}$ indicates identical residue, : indicates conserved substitutions, . indicates weakly similar substitutions. Colors indicate residues are classified into groups according to their physicochemical properties. Red: Nonpolar side chain; Green: Polar side chain; Blue: Negatively charged side chain; Magenta: Positively charged side chain.

\section{Abbreviations}

D. melanogaster or Dm: Drosophila melanogaster; T. castaneum or Tc: Tribolium castaneum; D. maculatus or Dmac: Dermestes maculatus; PRG: pair-rule gene; prd: paired; eve: even-skipped; COl: cytochrome c oxidase subunit I; bp: base pair; h: hour(s); AEL: after egg laying; gsb: gooseberry; gsb-n: gooseberry-neuro; PD: paired domain; HD: homeodomain; OP: octapeptide; SAZ: segment addition zone; vf: ventral furrow; hl: head lobe; paf: posterior amniotic fold; sw: serosal window; eRNAi: embryonic RNA interference; pRNAi: parental RNA interference; dsRNA: double-stranded RNA; en/En: engrailed/Engrailed; ps: parasegment.

\section{Authors' contributions}

$L P$ conceived of the study, designed the experiment, helped analyze the data, and drafted the manuscript. JX performed the DNA barcoding, SYTOX Green nuclear staining, Dmac-prd isolation and verification, in situ hybridization and RNAi. JX also analyzed the data and drafted the manuscript. ISF carried out the DAPI and phalloidin staining and helped to revise the manuscript. All authors read and approved the final manuscript.

\section{Author details}

${ }^{1}$ Department of Entomology, University of Maryland, 4112 Plant Sciences Building, College Park, MD 20742, USA. ${ }^{2}$ Program in Molecular and Cell Biology, University of Maryland, 4112 Plant Sciences Building, College Park, MD 20742, USA.

\section{Acknowledgements}

This work was supported by the National Science Foundation (IOS0950765) and the National Institutes of Health (GM113230). We thank Jeff Shultz for guidance throughout the years, Leo Shapiro for suggesting barcoding our species, Alison Heffer for preliminary experiments, and Alys Jarvela, Patricia Graham, Amanda Field and Katie Reding for comments on the manuscript. We also thank Amy Beaven and the Imaging Core at the University of Maryland for confocal microscopy support.

\section{Competing interests}

The authors declare that they have no competing interests.

Received: 16 June 2015 Accepted: 2 October 2015

Published online: 16 October 2015

\section{References}

1. Cheatle Jarvela AM, Pick L. Evo-Devo: discovery of diverse mechanisms. regulating development. 50th anniversary volume of current topics in developmental biology. 2015 (in press).

2. Zhang Z-Q. Animal biodiversity: an introduction to higher-level classification and taxonomic richness. Zootaxa. 2011;3148:6.

3. Wangler MF, Yamamoto S, Bellen HJ. Fruit flies in biomedical research. Genetics. 2015;199(3):639-53.

4. Misof B, Liu S, Meusemann K, Peters RS, Donath A, Mayer C, Frandsen PB, Ware J, Flouri T, Beutel RG, et al. Phylogenomics resolves the timing and pattern of insect evolution. Science. 2014;346(6210):763-7.

5. Grimaldi D, Engel MS. Evolution of the insects. 1st ed. Cambridge: Cambridge University Press; 2005.

6. World Conservation Monitoring Centre. Global biodiversity: status of the Earth's living resources. 1st ed. London: Chapman \& Hall; 1992.

7. Bouchard P, Grebennikov V, Smith ABT, Douglas H. Biodiversity of Coleoptera. USA: Blackwell Publishing; 2009

8. Hunt T, Bergsten J, Levkanicova Z, Papadopoulou A, John OS, Wild R, Hammond PM, Ahrens D, Balke M, Caterino MS, et al. A comprehensive phylogeny of beetles reveals the evolutionary origins of a superradiation. Science. 2007;318(5858):1913-6.

9. Brown SJ, Shippy TD, Miller S, Bolognesi R, Beeman RW, Lorenzen MD, Bucher G, Wimmer EA, Klingler M. The red flour beetle, Tribolium castaneum (Coleoptera): a model for studies of development and pest biology. Cold Spring Harb Protoc. 2009;2009(8):pdb.emo126.

10. Denell R. Establishment of Tribolium as a genetic model system and its early contributions to evo-devo. Genetics. 2008;180(4):1779-86.

11. Richards S, Gibbs RA, Weinstock GM, Brown SJ, Denell R, Beeman RW, Gibbs R, Bucher G, Friedrich M, Grimmelikhuijzen CJ, et al. The genome of the model beetle and pest Tribolium castaneum. Nature. 2008;452(7190):949-55.

12. Blair SS. Segmentation in animals. Curr Biol. 2008;18(21):R991-5.

13. Janssen R, Budd GE, Prpic NM, Damen WG. Expression of myriapod pair rule gene orthologs. Evodevo. 2011;2(1):5.

14. Janssen R, Budd GE. Deciphering the onychophoran 'segmentation gene cascade': gene expression reveals limited involvement of pair rule gene orthologs in segmentation, but a highly conserved segment polarity gene network. Dev Biol. 2013;382(1):224-34.

15. Gabriel WN, Goldstein B. Segmental expression of Pax3/7 and Engrailed homologs in tardigrade development. Dev Genes Evol. 2007;217(6):421-33.

16. Schoppmeier M, Damen WG. Expression of Pax group III genes suggests a single-segmental periodicity for opisthosomal segment patterning in the spider Cupiennius salei. Evol Dev. 2005;7(2):160-9.

17. Davis GK, Patel NH. Short, long, and beyond: molecular and embryological approaches to insect segmentation. Annu Rev Entomol. 2002;47:669-99. 
18. Rosenberg MI, Lynch JA, Desplan C. Heads and tails: evolution of antero-posterior patterning in insects. Biochim Biophys Acta. 2009;1789(4):333-42.

19. Peel AD, Chipman AD, Akam M. Arthropod segmentation: beyond the Drosophila paradigm. Nat Rev Genet. 2005;6(12):905-16.

20. Krause G. Die Eitypen der Insekten. Biol Zentralbl. 1939;59:495-536.

21. Liu PZ, Kaufman TC. Short and long germ segmentation: unanswered questions in the evolution of a developmental mode. Evol Dev. 2005;7(6):629-46.

22. Rosenberg MI, Brent AE, Payre F, Desplan C. Dual mode of embryonic development is highlighted by expression and function of Nasonia pairrule genes. Elife. 2014;3:e01440.

23. El-Sherif E, Averof M, Brown SJ. A segmentation clock operating in blastoderm and germband stages of Tribolium development. Development. 2012;139(23):4341-6.

24. Sarrazin AF, Peel AD, Averof M. A segmentation clock with two-segment periodicity in insects. Science. 2012;336(6079):338-41.

25. Choe CP, Miller SC, Brown SJ. A pair-rule gene circuit defines segments sequentially in the short-germ insect Tribolium castaneum. Proc Natl Acad Sci USA. 2006;103(17):6560-4.

26. Choe CP, Brown SJ. Evolutionary flexibility of pair-rule patterning revealed by functional analysis of secondary pair-rule genes, paired and sloppy-paired in the short-germ insect, Tribolium castaneum. Dev Biol. 2007;302(1):281-94.

27. Heffer A, Grubbs N, Mahaffey J, Pick L. The evolving role of the orphan nuclear receptor $f t z-f 1$, a pair-rule segmentation gene. Evol Dev. 2013;15(6):406-17.

28. Stuart JJ, Brown SJ, Beeman RW, Denell RE. A deficiency of the homeotic complex of the beetle Tribolium. Nature. 1991;350(6313):72-4.

29. Erezyilmaz DF, Kelstrup HC, Riddiford LM. The nuclear receptor E75A has a novel pair-rule-like function in patterning the milkweed bug, Oncopeltus fasciatus. Dev Biol. 2009;334(1):300-10.

30. Wilson MJ, Dearden PK. Pair-rule gene orthologues have unexpected maternal roles in the honeybee (Apis mellifera). PLoS One. 2012;7(9):e46490.

31. Sander K. Specification of the basic body pattern in insect embryogenesis. Adv Insect Physiol. 1976;12:125-238.

32. Patel NH, Condron BG, Zinn K. Pair-rule expression patterns of evenskipped are found in both short- and long-germ beetles. Nature. 1994;367(6462):429-34.

33. Hedges SB, Marin J, Suleski M, Paymer M, Kumar S. Tree of life reveals clock-like speciation and diversification. Mol Biol Evol. 2015;32(4):835-45.

34. Folmer O, Black M, Hoeh W, Lutz R, Vrijenhoek R. DNA primers for amplification of mitochondrial cytochrome c oxidase subunit I from diverse metazoan invertebrates. Mol Mar Biol Biotechnol. 1994;3(5):294-9.

35. Bely AE, Weisblat DA. Lessons from leeches: a call for DNA barcoding in the lab. Evol Dev. 2006;8(6):491-501.

36. Kosman D, Mizutani CM, Lemons D, Cox WG, McGinnis W, Bier E. Multiplex detection of RNA expression in Drosophila embryos. Science. 2004;305(5685):846

37. Liu P, Kaufman TC. Dissection and fixation of large milkweed bug (Oncopeltus) embryos. Cold Spring Harb Protoc. 2009;2009(8):pdb.prot5261.

38. Davis GK, Jaramillo CA, Patel NH. Pax group III genes and the evolution of insect pair-rule patterning. Development. 2001;128(18):3445-58.

39. Bopp D, Burri M, Baumgartner S, Frigerio G, Noll M. Conservation of a large protein domain in the segmentation gene paired and in functionally related genes of Drosophila. Cell. 1986;47(6):1033-40.

40. Baumgartner S, Bopp D, Burri M, Noll M. Structure of two genes at the gooseberry locus related to the paired gene and their spatial expression during Drosophila embryogenesis. Genes Dev. 1987;1(10):1247-67.

41. Gutjahr T, Patel NH, Li X, Goodman CS, Noll M. Analysis of the gooseberry locus in Drosophila embryos: gooseberry determines the cuticular pattern and activates gooseberry neuro. Development. 1993;118(1):21-31.

42. Ramos RG, Machado LC, Moda LM. Fluorescent visualization of macromolecules in Drosophila whole mounts. Methods Mol Biol. 2010;588:165-79.

43. Kosman D, Small S. Concentration-dependent patterning by an ectopic expression domain of the Drosophila gap gene knirps. Development. 1997;124(7):1343-54.
44. Gutjahr T, Vanario-Alonso CE, Pick L, Noll M. Multiple regulatory elements direct the complex expression pattern of the Drosophila segmentation gene paired. Mech Dev. 1994;48(2):119-28.

45. Nagaso H, Murata T, Day N, Yokoyama KK. Simultaneous detection of RNA and protein by in situ hybridization and immunological staining. J Histochem Cytochem. 2001;49(9):1177-82.

46. Gilbert SF. Developmental Biology. 9th ed. Sunderland: Sinauer Associates, Inc.; 2010.

47. Foe VE, Odell GM, Edgar BA. Mitosis and Morphogenesis in the Drosophila Embryo: point and counterpoint. In: Bate M, Hartenstein V, editors. The development of Drosophila melanogaster. Plainview: Cold Spring Harbor Lab. Press; 1993. p. 149-300.

48. Frigerio G, Burri M, Bopp D, Baumgartner S, Noll M. Structure of the segmentation gene paired and the Drosophila PRD gene set as part of a gene network. Cell. 1986;47(5):735-46.

49. Burri M, Tromvoukis Y, Bopp D, Frigerio G, Noll M. Conservation of the paired domain in metazoans and its structure in three isolated human genes. EMBO J. 1989;8(4):1183-90.

50. Keller RG, Desplan C, Rosenberg MI. Identification and characterization of Nasonia Pax genes. Insect Mol Biol. 2010;19(Suppl 1):109-20.

51. Noll M. Evolution and role of Pax genes. Curr Opin Genet Dev. 1993:3(4):595-605.

52. Treisman J, Gonczy P, Vashishtha M, Harris E, Desplan C. A single amino acid can determine the DNA binding specificity of homeodomain proteins. Cell. 1989;59(3):553-62.

53. Gutjahr T, Frei E, Noll M. Complex regulation of early paired expression: initial activation by gap genes and pattern modulation by pair-rule genes. Development. 1993;117(2):609-23.

54. Kilchherr F, Baumgartner S, Bopp D, Frei E, Noll M. Isolation of the paired gene of Drosophila and its spatial expression during early embryogenesis. Nature. 1986;321(29):493-9.

55. Osborne PW, Dearden PK. Expression of Pax group //I genes in the honeybee (Apis mellifera). Dev Genes Evol. 2005;215(10):499-508.

56. Mito T, Kobayashi C, Sarashina I, Zhang H, Shinahara W, Miyawaki K, Shinmyo Y, Ohuchi H, Noji S. even-skipped has gap-like, pair-rule-like, and segmental functions in the cricket Gryllus bimaculatus, a basal, intermediate germ insect (Orthoptera). Dev Biol. 2007;303(1):202-13.

57. Lynch JA, Brent AE, Leaf DS, Pultz MA, Desplan C. Localized maternal orthodenticle patterns anterior and posterior in the long germ wasp Nasonia. Nature. 2006;439(7077):728-32.

58. Bucher G, Scholten J, Klingler M. Parental RNAi in Tribolium (Coleoptera). Curr Biol. 2002;12(3):R85-6.

59. Mito T, Sarashina I, Zhang H, Iwahashi A, Okamoto H, Miyawaki K, Shinmyo Y, Ohuchi H, Noji S. Non-canonical functions of hunchback in segment patterning of the intermediate germ cricket Gryllus bimaculatus. Development. 2005;132(9):2069-79.

60. Liu PZ, Kaufman TC. hunchback is required for suppression of abdominal identity, and for proper germband growth and segmentation in the intermediate germband insect Oncopeltus fasciatus. Development. 2004;131(7):1515-27.

61. Ciudad L, Piulachs MD, Bellés X. Systemic RNAi of the cockroach vitellogenin receptor results in a phenotype similar to that of the Drosophila yolkless mutant. FEBS J. 2006;273(2):325-35.

62. DiNardo $\mathrm{S}, \mathrm{O}^{\prime}$ Farrell PH. Establishment and refinement of segmental pattern in the Drosophila embryo: spatial control of engrailed expression by pair-rule genes. Genes Dev. 1987;1(10):1212-25.

63. Nüsslein-Volhard C, Wieschaus E. Mutations affecting segment number and polarity in Drosophila. Nature. 1980;287(5785):795-801.

64. Maderspacher F, Bucher G, Klingler M. Pair-rule and gap gene mutants in the flour beetle Tribolium castaneum. Dev Genes Evol. 1998;208(10):558-68.

65. Sommer RJ, Tautz D. Involvement of an orthologue of the Drosophila pairrule gene hairy in segment formation of the short germ-band embryo of Tribolium (Coleoptera). Nature. 1993:361(6411):448-50.

66. Brown SJ, Patel NH, Denell RE. Embryonic expression of the single Tribolium engrailed homolog. Dev Genet. 1994;15(1):7-18.

67. Nagy LM, Carroll S. Conservation of wingless patterning functions in the short-germ embryos of Tribolium castaneum. Nature. 1994;367(6462):460-3. 
68. Graves R. Beetles \& bones: care, feeding, and use of dermestid beetles. South Berwick, ME; Jillett Publications; 2005.

69. Shaver B, Kaufman PE. Common name: hide beetle. 2009. http://entnemdept.ufl.edu/creatures/misc/beetles/hide_beetle.htm. Accessed 10 Nov 2012.

70. Veer V, Negi BK, Rao KM. Dermestid beetles and some other insect pests associated with stored silkworm cocoons in India, including a world list of dermestid species found attacking this commodity. J Stored Prod Res 1996:32(1):69-89.

71. Gullan PJ, Cranston PS. The insects: an outline of Entomology. 4th ed. Oxford: Blackwell Publishing; 2010.

72. Huvenne H, Smagghe G. Mechanisms of dsRNA uptake in insects and potential of RNAi for pest control: a review. J Insect Physiol. 2010;56(3):227-35.

73. Carroll SB, Grenier JK, Weatherbee SD. From DNA to diversity: molecular genetics and the evolution of animal design. 2nd ed. Malden: Blackwell Publishing; 2005.

74. Degnan BM, Vervoort M, Larroux C, Richards GS. Early evolution of metazoan transcription factors. Curr Opin Genet Dev. 2009;19(6):591-9.

75. Wang Q, Fang WH, Krupinski J, Kumar S, Slevin M, Kumar P. Pax genes in embryogenesis and oncogenesis. J Cell Mol Med. 2008;12(6A):2281-94.

76. Buckingham M, Relaix F. The role of Pax genes in the development of tissues and organs: Pax3 and Pax7 regulate muscle progenitor cell functions. Annu Rev Cell Dev Biol. 2007;23:645-73.

77. Jun S, Desplan C. Cooperative interactions between paired domain and homeodomain. Development. 1996;122(9):2639-50.

78. Bopp D, Jamet E, Baumgartner S, Burri M, Noll M. Isolation of two tissuespecific Drosophila paired box genes, Pox meso and Pox neuro. EMBO J. 1989:8(11):3447-57.

79. Underhill DA. PAX proteins and fables of their reconstruction. Crit Rev Eukaryot Gene Expr. 2012;22(2):161-77.

80. Breitling R, Gerber JK. Origin of the paired domain. Dev Genes Evol. 2000;210(12):644-50.
81. Paixão-Côrtes VR, Salzano FM, Bortolini MC. Origins and evolvability of the PAX family. Semin Cell Dev Biol. 2015. doi:10.1016/j.semcdb.2015.08.014.

82. Friedrich M. Evo-Devo gene toolkit update: at least seven Pax transcription factor subfamilies in the last common ancestor of bilaterian animals. Evol Dev. 2015;17(5):255-7.

83. Li X, Noll M. Role of the gooseberry gene in Drosophila embryos: maintenance of wingless expression by a wingless-gooseberry autoregulatory loop. EMBO J. 1993;12(12):4499-509.

84. Stuart ET, Kioussi C, Gruss P. Mammalian Pax genes. Annu Rev Genet. 1994;28:219-36.

85. Green J, Akam M. Evolution of the pair rule gene network: insights from a centipede. Dev Biol. 2013;382(1):235-45.

86. Davis GK, D'Alessio JA, Patel NH. Pax3/7 genes reveal conservation and divergence in the arthropod segmentation hierarchy. Dev Biol. 2005;285(1):169-84.

87. Franke FA, Schumann I, Hering L, Mayer G. Phylogenetic analysis and expression patterns of Pax genes in the onychophoran Euperipatoides rowelli reveal a novel bilaterian Pax subfamily. Evol Dev. 2015;17(1):3-20.

88. Janssen R, Damen WG, Budd GE. Expression of pair rule gene orthologs in the blastoderm of a myriapod: evidence for pair rule-like mechanisms? BMC Dev Biol. 2012;12:15.

89. Dearden PK, Donly C, Grbić M. Expression of pair-rule gene homologues in a chelicerate: early patterning of the two-spotted spider mite Tetranychus urticae. Development. 2002;129(23):5461-72.

90. Aranda M, Marques-Souza H, Bayer T, Tautz D. The role of the segmentation gene hairy in Tribolium. Dev Genes Evol. 2008;218(9):465-77.

91. Vanario-Alonso CE, O'Hara E, McGinnis W, Pick L. Targeted ribozymes reveal a conserved function of the Drosophila paired gene in sensory organ development. Mech Dev. 1995;53(3):323-8.

92. Coulter DE, Wieschaus E. Gene activities and segmental patterning in Drosophila: analysis of odd-skipped and pair-rule double mutants. Genes Dev. 1988;2(12B):1812-23.

\section{Submit your next manuscript to BioMed Central and take full advantage of:}

- Convenient online submission

- Thorough peer review

- No space constraints or color figure charges

- Immediate publication on acceptance

- Inclusion in PubMed, CAS, Scopus and Google Scholar

- Research which is freely available for redistribution

Submit your manuscript at

www.biomedcentral.com/submit

C BioMed Central 